\title{
Hierarchical quantum communication
}

June 10, 2018

\author{
Chitra Shukla ${ }^{a}$ and Anirban Pathak $\left.{ }^{a, b}, 1\right]$ \\ ${ }^{a}$ Department of Physics and Materials Science Engineering, \\ Jaypee Institute of Information Technology, A-10, Sector-62, Noida, UP-201307, India. \\ ${ }^{b}$ RCPTM, Joint Laboratory of Optics of Palacky University and Institute of Physics of Academy of \\ Science of the Czech Republic, Faculty of Science, Palacky University, 17. listopadu 12, 77146 Olomouc, \\ Czech Republic.
}

\begin{abstract}
A general approach to study the hierarchical quantum information splitting (HQIS) is proposed and the same is used to systematically investigate the possibility of realizing HQIS using different classes of 4-qubit entangled states that are not connected by SLOCC. Explicit examples of HQIS using 4-qubit cluster state and 4-qubit $|\Omega\rangle$ state are provided. Further, the proposed HQIS scheme is generalized to introduce two new aspects of hierarchical quantum communication. To be precise, schemes of probabilistic hierarchical quantum information splitting and hierarchical quantum secret sharing are obtained by modifying the proposed HQIS scheme. A number of practical situations where hierarchical quantum communication would be of use are also presented.
\end{abstract}

PACS: 03.67.Dd, 03.67.Hk, 03.65.Yz

Keywords: Hierarchical quantum communication, quantum information splitting, quantum secret sharing.

\section{Introduction}

In 1993 Bennett, Brassard, Crépeau, Jozsa, Peres and Wootters [1] proposed a scheme for quantum teleportation using Bell state. This scheme drew considerable attention of the quantum communication community as the teleportation has no classical analog. Since the pioneering work of Bennett et al. a large number of teleportation schemes and their applications have been reported [1, 2, 3, 4, 15, 6, 17, 8, 9, 10, 11, 12, 13, 14]. These teleportation schemes can be primarily classified into two broad classes: a) Perfect teleportation schemes [1, 2] and b) Probabilistic teleportation schemes [3, 4, 5, 6]. By perfect teleportation we mean that the success rate of teleportation is unity. This requires a maximally entangled quantum channel. However, teleportation with unit fidelity is possible even when the quantum channel is non-maximally entangled. In that case the success rate of the receiver is not unity and the teleportation scheme is referred to as the probabilistic teleportation scheme. Further, the teleportation schemes are not limited to two-party teleportation (i.e., teleportation between Alice and Bob). Many schemes of multi-party quantum teleportation

\footnotetext{
1email: anirban.pathak@gmail.com, phone: +420608650694
} 
are also proposed in the last two decades ([2, 7] and references therein). Such multi-party teleportation schemes have led to a set of interesting applications. Probably the most interesting and most fundamental multi-party quantum teleportation scheme is the controlled teleportation (CT) or the quantum information splitting (QIS) scheme [2, 7]. In this scheme, Alice shares prior entanglement with Bob and at least one Charlie (supervisor). Now nocloning theorem ensures that Alice cannot teleport copies of the unknown quantum state (qubit) to both Charlie and Bob. Consequently, if Alice succeeds to teleport an unknown quantum state to Charlie and Bob then only one of them will be able to reconstruct the state with the help of the other. Thus the quantum information is split among Charlie and Bob and this is why such a scheme is called QIS scheme. Now usually we assume that Bob recovers the unknown state with the help of Charlie. Keeping this in mind, often Charlie is referred to as the supervisor. Since Charlie can control the teleportation protocol, this type of protocol is often referred to as CT protocol too. Thus CT schemes are equivalent to QIS schemes. In recent past CT/QIS schemes are reported using GHZ-like state [2], cluster state [7], W state [8], etc.

Till recent past all the studies on multi-party quantum teleportation were restricted to symmetric quantum information splitting where all the receivers were equally capable to recover the unknown quantum state sent by the sender (Alice). Recently the concept of asymmetric quantum information splitting is introduced by Wang et al. [9, 10, 11. In their scheme Boss (Alice) distributes a quantum state among several agents who are spatially separated. The agents are graded in accordance to their power to recover the quantum state sent by Alice. A high power agent does not require the help of all other agents to reconstruct the quantum state sent by Alice, whereas a low power agent can reconstruct the secret state iff all other agents cooperate with him. Thus there is a hierarchy among the agents and consequently, the scheme is referred to as the hierarchical quantum information splitting (HQIS) scheme. Wang et al. have recently reported HQIS using 4-qubit $|\chi\rangle$ state [9], $t$-qubit graph state in general where $t \geq 3$ [10], and 6-qubit cluster state [11]. Extending their idea here we propose a systematic and general procedure to investigate the possibility of HQIS using an arbitrary $(n+1)$-qubit entangled state and use our approach to explicitly show that the HQIS is possible with 4-qubit $|\Omega\rangle$ state and 4-qubit cluster state.

There exist several variants and applications of QIS. For example one may think of probabilistic QIS or of Hillery, Buzek and Bertaiume's [12] idea of quantum secret sharing (QSS) which may be viewed as an application of QIS. So far all the schemes proposed for QSS and probabilistic QIS are symmetric. This observation motivated us to investigate the possibilities of introducing asymmetry (hierarchy) among the receivers involved in a QSS or probabilistic QIS scheme. The same is done here and that lead to the first ever protocols of probabilistic HQIS and hierarchical QSS (HQSS). The proposed schemes are interesting for several reasons. Primarily they are interesting for their relevance in many practical situations. For example, we may consider that Alice is president of a country and Diana is the defense minister of that country. Bob and Charlie are defense secretary and chief of the armed forces of that country, respectively. Now if the president wishes to permit the use of a nuclear weapon at a suitable time then she distributes an information (say, a code which is required to unlock the nuclear weapon) among the defense minister, the defense secretary and the chief of the armed forces in such a way that the minister can unlock the weapon if either the defense secretary or the chief of the armed forces agrees and cooperates with him. However, if the chief of the armed forces or the defense secretary wants to unlock the weapon they would require the cooperation of each other and that of the defense minister. Thus the defense minister is more powerful than the chief of the armed forces and the defense secretary, but even she is not powerful enough to unlock the weapon alone. To realize this practically relevant scenario with unconditional security we would require a scheme for HQSS. In what follows we will see that HQSS using $|\Omega\rangle$ can be used to realize this scenario. Here it would be apt to note that any attempt to implement the above scenario using classical resources will not be unconditionally secure. Another sector where HQSS is of everyday need is banking, where a bank manager and/or cashier is usually more powerful than the other users (office assistants and secretaries). However, even the bank manager alone is not powerful enough to perform all the financial 
operations related to his bank. For example, the password required to unlock an ATM is always split into two or more pieces and the manager alone cannot unlock it. Similarly, hierarchical secret sharing is also essential for the smooth operation of the departmental stores. The proposed schemes are also important because the existence of HQIS automatically implies the existence of many related aspects of controlled teleportation (e.g. controlled quantum information splitting, controlled quantum secret sharing, controlled quantum state sharing etc.).

Remaining part of the paper is organized as follows. In Section 2 we have described a generalized approach to investigate the possibility of HQIS using $(n+1)$-qubit entangled states in general and as examples we have explicitly shown that HQIS is possible using 4-qubit $|\Omega\rangle$ state and 4-qubit cluster state. In Section 3 we have modified our scheme to obtain a scheme for probabilistic HQIS. The HQIS scheme is further generalized in Section 4 to yield a scheme of HQSS. Finally, Section 5 is dedicated for the conclusions.

\section{A generalized approach to perfect HQIS}

Let us start with a general $(n+1)$ qubit state of the form

$$
\left|\psi_{c}\right\rangle=\frac{1}{\sqrt{2}}\left[|0\rangle\left|\psi_{0}\right\rangle+|1\rangle\left|\psi_{1}\right\rangle\right]
$$

where $\left|\psi_{0}\right\rangle$ and $\left|\psi_{1}\right\rangle$ are arbitrary $n$ qubit state. Any arbitrary quantum state can be written in the above form but for our purpose we would choose those states where $\left|\psi_{0}\right\rangle$ and $\left|\psi_{1}\right\rangle$ are orthogonal to each other 2 . The subscript $c$ stands for channel. We consider that the first qubit of $\left|\psi_{\mathbf{c}}\right\rangle$ is with Alice and the rest are with $n$ agents, say Bob ${ }_{1}, B_{1} b_{2}$ etc. If we work with $n=3$, we may call the agents as Bob, Charlie and Diana and represent their respective qubits by the subscripts $B, C, D$. Similarly, the qubit of Alice is indexed by the subscript $A$. So Alice shares $\left|\psi_{c}\right\rangle$ with $n$ agents and each agent has one qubit.

Consider that Alice wishes to teleport (share) among her agents a general one qubit state

$$
\left|\psi_{s}\right\rangle=\frac{1}{\sqrt{1+|\lambda|^{2}}}(|0\rangle+\lambda|1\rangle)
$$

which represents an arbitrary qubit. So the combined state of Alice and her agents is

$$
\begin{aligned}
\left|\psi_{s}\right\rangle \otimes\left|\psi_{c}\right\rangle & =\frac{1}{\sqrt{1+|\lambda|^{2}}}(|0\rangle+\lambda|1\rangle) \otimes \frac{1}{\sqrt{2}}\left[|0\rangle\left|\psi_{0}\right\rangle+|1\rangle\left|\psi_{1}\right\rangle\right] \\
& =\frac{1}{\sqrt{2\left(1+|\lambda|^{2}\right)}}\left[|00\rangle\left|\psi_{0}\right\rangle+|01\rangle\left|\psi_{1}\right\rangle\right]+\frac{\lambda}{\sqrt{2\left(1+|\lambda|^{2}\right)}}\left[|10\rangle\left|\psi_{0}\right\rangle+|11\rangle\left|\psi_{1}\right\rangle\right] \\
& =\frac{1}{2 \sqrt{\left(1+|\lambda|^{2}\right)}}\left[\left|\psi^{+}\right\rangle\left(\left|\psi_{0}\right\rangle+\lambda\left|\psi_{1}\right\rangle\right)+\left|\psi^{-}\right\rangle\left(\left|\psi_{0}\right\rangle-\lambda\left|\psi_{1}\right\rangle\right)\right. \\
& \left.+\left|\phi^{+}\right\rangle\left(\left|\psi_{1}\right\rangle+\lambda\left|\psi_{0}\right\rangle\right)+\left|\phi^{-}\right\rangle\left(\left|\psi_{1}\right\rangle-\lambda\left|\psi_{0}\right\rangle\right)\right] .
\end{aligned}
$$

Now Alice does a Bell measurement on the first 2 qubits. From (3) we can see that after the Bell measurement of Alice the combined states of all the $n$ agents reduces to $\left|\Psi^{ \pm}\right\rangle=\frac{\left|\psi_{0}\right\rangle \pm \lambda\left|\psi_{1}\right\rangle}{\sqrt{1+|\lambda|^{2}}}$ or $\left|\Phi^{ \pm}\right\rangle=\frac{\left|\psi_{1}\right\rangle \pm \lambda\left|\psi_{0}\right\rangle}{\sqrt{1+|\lambda|^{2}}}$. To be precise, the complete relation between the outcome of the Bell measurement of Alice and the combined state of the agents is given in Table 1, which is true in general. This provides us the basic framework to investigate the possibilities of HQIS in different quantum states. The remaining task is to appropriately decompose the combined state of the agents so that one of them can recover the unknown state and to find out the appropriate unitary operation. In the following subsections we will consider two specific cases and explicitly show that the above framework can be used to establish that HQIS is possible with 4-qubit $|\Omega\rangle$ state and 4-qubit cluster state.

\footnotetext{
${ }^{2}$ This choice would ensure that $\left|\psi_{\mathbf{c}}\right\rangle$ is an entangled state.
} 


\begin{tabular}{|c|c|}
\hline $\begin{array}{c}\text { Outcome of Alice's } \\
\text { measurement }\end{array}$ & $\begin{array}{c}\text { Combined state of all } \\
\text { agents after the } \\
\text { measurement of Alice }\end{array}$ \\
\hline$\left|\psi^{+}\right\rangle$ & $\left|\Psi^{+}\right\rangle=\frac{\left|\psi_{0}\right\rangle+\lambda\left|\psi_{1}\right\rangle}{\sqrt{1+|\lambda|^{2}}}$ \\
\hline$\left|\psi^{-}\right\rangle$ & $\left|\Psi^{-}\right\rangle=\frac{\left|\psi_{0}\right\rangle-\lambda\left|\psi_{1}\right\rangle}{\sqrt{1+|\lambda|^{2}}}$ \\
\hline$\left|\phi^{+}\right\rangle$ & $\left|\Phi^{+}\right\rangle=\frac{\left|\psi_{1}\right\rangle+\lambda\left|\psi_{0}\right\rangle}{\sqrt{1+|\lambda|^{2}}}$ \\
\hline$\left|\phi^{-}\right\rangle$ & $\left|\Phi^{-}\right\rangle=\frac{\left|\psi_{1}\right\rangle-\lambda\left|\psi_{0}\right\rangle}{\sqrt{1+|\lambda|^{2}}}$ \\
\hline
\end{tabular}

Table 1: Relation between Alice's measurement outcomes and the combined states of the agents after the measurement of Alice.

\subsection{Case I: $\left|\psi_{c}\right\rangle$ is 4-qubit $|\Omega\rangle$ state}

Let us assume that Alice has chosen 4-qubit $|\Omega\rangle$ state [15] as channel and kept the first photon with her and has sent the second, third and fourth photons to Bob, Charlie and Diana respectively. In that case

$$
\begin{aligned}
\left|\psi_{c}\right\rangle=|\Omega\rangle_{A B C D} & =\frac{1}{2}[|0000\rangle+|0110\rangle+|1001\rangle-|1111\rangle]_{A B C D} \\
& =\frac{1}{\sqrt{2}}\left[|0\rangle_{A}\left|\psi_{0}\right\rangle_{B C D}+|1\rangle_{A}\left|\psi_{1}\right\rangle_{B C D}\right],
\end{aligned}
$$

where $\left|\psi_{0}\right\rangle_{B C D}=\frac{1}{\sqrt{2}}[|000\rangle+|110\rangle]$ and $\left|\psi_{1}\right\rangle_{B C D}=\frac{1}{\sqrt{2}}[|001\rangle-|111\rangle]$.

Now after Alice's Bell measurement on the first two qubits, the combined state of Bob, Charlie and Diana collapses according to Table 1. If Alice's measurement outcome is $\left|\psi^{ \pm}\right\rangle$then the state of the agents is

$$
\begin{aligned}
\left|\Psi^{ \pm}\right\rangle_{B C D} & =\frac{1}{\sqrt{1+|\lambda|^{2}}}\left[\left|\psi_{0}\right\rangle_{B C D} \pm \lambda\left|\psi_{1}\right\rangle_{B C D}\right] \\
& =\frac{1}{\sqrt{2\left(1+|\lambda|^{2}\right)}}[|000\rangle+|110\rangle \pm \lambda(|001\rangle-|111\rangle)]_{B C D} .
\end{aligned}
$$

Similarly, if Alice obtains $\left|\phi^{ \pm}\right\rangle$then the state of the agents is

$$
\begin{aligned}
\left|\Phi^{ \pm}\right\rangle_{B C D} & =\frac{1}{\sqrt{1+|\lambda|^{2}}}\left[\left|\psi_{1}\right\rangle_{B C D} \pm \lambda\left|\psi_{0}\right\rangle_{B C D}\right] \\
& =\frac{1}{\sqrt{2\left(1+|\lambda|^{2}\right)}}[|001\rangle-|111\rangle \pm \lambda(|000\rangle+|110\rangle)]_{B C D}
\end{aligned}
$$

Now if the agents decide that Diana will recover the quantum state sent by Alice, then we can decompose (5) and (6) as

$$
\left|\Psi^{ \pm}\right\rangle_{B C D}=\frac{1}{\sqrt{2\left(1+|\lambda|^{2}\right)}}\left[|00\rangle_{B C}\left(|0\rangle_{D} \pm \lambda|1\rangle_{D}\right)+|11\rangle_{B C}\left(|0\rangle_{D} \mp \lambda|1\rangle_{D}\right)\right]
$$

and

$$
\left|\Phi^{ \pm}\right\rangle_{B C D}=\frac{1}{\sqrt{2\left(1+|\lambda|^{2}\right)}}\left[|00\rangle_{B C}\left(|1\rangle_{D} \pm \lambda|0\rangle_{D}\right)-|11\rangle_{B C}\left(|1\rangle_{D} \mp \lambda|0\rangle_{D}\right)\right] .
$$

Now form (7) and (8) it is clear that if Bob (Charlie) measures his (her) qubit in the computational basis and sends the result to Diana, then Diana will be able to reconstruct the state sent by Alice using appropriate unitary operators as shown in Table 2, For example, if Alice's measurement outcome is $\left|\psi^{+}\right\rangle$ and that of Charlie is $|0\rangle$ then the state of Diana is collapsed to $\frac{1}{\sqrt{1+|\lambda|^{2}}}(|0\rangle+\lambda|1\rangle)$, so Diana needs to apply $I$. Thus Diana needs the help of Alice and either Charlie or Bob to reconstruct the unknown state 


\begin{tabular}{|c|c|c|}
\hline Alice measurement outcome & Bob, Charlie measurement outcome & Diana's operation \\
\hline$\left|\psi^{+}\right\rangle$ & $|00\rangle_{B C}$ & $I$ \\
\hline$\left|\psi^{+}\right\rangle$ & $|11\rangle_{B C}$ & $Z$ \\
\hline$\left|\psi^{-}\right\rangle$ & $|00\rangle_{B C}$ & $Z$ \\
\hline$\left|\psi^{-}\right\rangle$ & $|11\rangle_{B C}$ & $I$ \\
\hline$\left|\phi^{+}\right\rangle$ & $|00\rangle_{B C}$ & $X$ \\
\hline$\left|\phi^{+}\right\rangle$ & $|11\rangle_{B C}$ & $X Z$ \\
\hline$\left|\phi^{-}\right\rangle$ & $|00\rangle_{B C}$ & $X Z$ \\
\hline$\left|\phi^{-}\right\rangle$ & $|11\rangle_{B C}$ & $X$ \\
\hline
\end{tabular}

Table 2: Relation among the measurement outcomes of Alice, Bob and Charlie and the unitary operations to be applied by Diana when the initial state is an $|\Omega\rangle$ state and Diana reconstructs the unknown state sent by Alice. Here the measurement outcomes of Bob and Charlie are always same. So the communication from one of them and Alice would be sufficient for Diana to reconstruct the unknown state sent by Alice.

sent by Alice. Now we would like to ask what happens if the agents decide that Bob will reconstruct the state sent by Alice. From (5) and (6) it is clear that Charlie and Diana cannot measure their qubits in the computational basis as that would collapse the state of Bob to $|0\rangle$ or $|1\rangle$. Further, we note that we can also decompose (15) and (6) as

$$
\begin{aligned}
\left|\Psi^{ \pm}\right\rangle_{B C D} & =\frac{1}{2 \sqrt{\left(1+|\lambda|^{2}\right)}}\left[\left(|0\rangle_{B} \mp \lambda|1\rangle_{B}\right)\left|\psi^{+}\right\rangle_{C D}+\left(|0\rangle_{B} \pm \lambda|1\rangle_{B}\right)\left|\psi^{-}\right\rangle_{C D}\right. \\
& \left.+\left(|1\rangle_{B} \pm \lambda|0\rangle_{B}\right)\left|\phi^{+}\right\rangle_{C D}-\left(|1\rangle_{B} \mp \lambda|0\rangle_{B}\right)\left|\phi^{-}\right\rangle_{C D}\right] \\
\left|\Phi^{ \pm}\right\rangle_{B C D} & =\frac{1}{2 \sqrt{\left(1+|\lambda|^{2}\right)}}\left[\left(|0\rangle_{B} \pm \lambda|1\rangle_{B}\right)\left|\phi^{+}\right\rangle_{C D}+\left(|0\rangle_{B} \mp \lambda|1\rangle_{B}\right)\left|\phi^{-}\right\rangle_{C D}\right. \\
& \left.-\left(|1\rangle_{B} \mp \lambda|0\rangle_{B}\right)\left|\psi^{+}\right\rangle_{C D}+\left(|1\rangle_{B} \pm \lambda|0\rangle_{B}\right)\left|\psi^{-}\right\rangle_{C D}\right] .
\end{aligned}
$$

From (9) and (10) we can observe that Bob can recover the arbitrary state $\left|\psi_{s}\right\rangle$ if a Bell measurement can be done on the qubits of Charlie and Diana. This can be fulfilled by either one of them (Charlie and Diana) communicating a qubit to the other over an authenticated quantum channel, or both performing a joint measurement (a nonlocal operation). The relation among the measurement outcomes of Alice, Charlie and Diana and the unitary operations to be applied by Bob to reconstruct the unknown quantum state is explicitly provided in the Table 3. Here Charlie and Diana need to perform a joint measurement and consequently Bob requires assistance of both of them and Alice to reconstruct the unknown state sent by Alice. Thus Bob requires more information than that required by Diana to reconstruct the unknown quantum state. Consequently, Diana is more powerful than Bob 3 and the scheme described here is a scheme of HQIS.

A special case of the above situation may be visualized as follows: Alice sends Both $\mathrm{C}$ and $\mathrm{D}$ qubits to $\mathrm{Bob}_{1}$ and qubit $\mathrm{B}$ to $\mathrm{Bob}_{2}$. Now Bob $\mathrm{b}_{1}$ can measure qubit $\mathrm{C}$ in computational basis and apply unitary operation in accordance with Table 2 to reconstruct the state sent by Alice. To do so he does not require any help of $\mathrm{Bob}_{2}$. However, Bob 2 can reconstruct the state iff Bob $\mathrm{b}_{1}$ measures his qubits in Bell basis and conveys the result to $\mathrm{Bob}_{2}$.

We may now note that the state with Bob in the first equation of (9) can be considered as quantum encrypted with classical data of 2 bits in the joint possession of Charlie and Diana, which is seen manifestly as follows.

$$
\left|\Psi^{+}\right\rangle_{B C D}=\frac{1}{2}\left[\left(Z\left|\psi_{s}\right\rangle\right)_{B}\left|\psi^{+}\right\rangle_{C D}+\left(I\left|\psi_{s}\right\rangle\right)_{B}\left|\psi^{-}\right\rangle_{C D}+\left(X\left|\psi_{s}\right\rangle\right)_{B}\left|\phi^{+}\right\rangle_{C D}-\left(i Y\left|\psi_{s}\right\rangle\right)_{B}\left|\phi^{-}\right\rangle_{C D}\right] .
$$

\footnotetext{
${ }^{3}$ Charlie and Bob are equally powerful here. As the state of Charlie and Bob are perfectly correlated, we have not explicitly described the case where Charlie recovers the unknown quantum state.
} 


\begin{tabular}{|c|c|c|}
\hline Alice measurement outcome & $\begin{array}{c}\text { Outcome of joint } \\
\text { measurement of Charlie and } \\
\text { Diana }\end{array}$ & Bob's operation \\
\hline$\left|\psi^{+}\right\rangle$ & $\left|\psi^{+}\right\rangle_{C D}$ & $Z$ \\
\hline$\left|\psi^{+}\right\rangle$ & $\left|\psi^{-}\right\rangle_{C D}$ & $I$ \\
\hline$\left|\psi^{+}\right\rangle$ & $\left|\phi^{+}\right\rangle_{C D}$ & $X$ \\
\hline$\left|\psi^{+}\right\rangle$ & $\left|\phi^{-}\right\rangle_{C D}$ & $I$ \\
\hline$\left|\psi^{-}\right\rangle$ & $\left|\psi^{+}\right\rangle_{C D}$ & $Z$ \\
\hline$\left|\psi^{-}\right\rangle$ & $\left|\psi^{-}\right\rangle_{C D}$ & $X Z$ \\
\hline$\left|\psi^{-}\right\rangle$ & $\left|\phi^{+}\right\rangle_{C D}$ & $X$ \\
\hline$\left|\psi^{-}\right\rangle$ & $\left|\phi^{-}\right\rangle_{C D}$ & $I$ \\
\hline$\left|\phi^{+}\right\rangle$ & $\left|\phi^{+}\right\rangle_{C D}$ & $X Z$ \\
\hline$\left|\phi^{+}\right\rangle$ & $\left|\phi^{-}\right\rangle_{C D}$ & $X$ \\
\hline$\left|\phi^{+}\right\rangle$ & $\left|\psi^{+}\right\rangle_{C D}$ & $Z$ \\
\hline$\left|\phi^{+}\right\rangle$ & $\left|\psi^{-}\right\rangle_{C D}$ & $X$ \\
\hline$\left|\phi^{-}\right\rangle$ & $\left|\phi^{+}\right\rangle_{C D}$ & $X Z$ \\
\hline$\left|\phi^{-}\right\rangle$ & $\left|\phi^{-}\right\rangle_{C D}$ & $\left|\psi^{+}\right\rangle_{C D}$ \\
\hline$\left|\phi^{-}\right\rangle$ & $\left|\psi^{-}\right\rangle_{C D}$ & \\
\hline$\left|\phi^{-}\right\rangle$ & & \\
\hline
\end{tabular}

Table 3: Relation among the measurement outcomes of Alice, Charlie and Diana and the unitary operations to be applied by Bob when the initial state is an $|\Omega\rangle$ state and Bob reconstructs the unknown state. Here Charlie and Diana needs to do a joint measurement and consequently Bob requires assistance of both of them and Alice to reconstruct the unknown state sent by Alice.

Without access to knowledge of the state with Charlie and Diana, Bob's state is given by the reduced density operator:

$$
\frac{1}{4}\left(\left|\psi_{s}\right\rangle\left\langle\psi_{s}|+Z| \psi_{s}\right\rangle\left\langle\psi_{s}|Z+X| \psi_{s}\right\rangle\left\langle\psi_{s}|X+i Y| \psi_{s}\right\rangle\left\langle\psi_{s}\right| i Y\right)=\frac{I}{2}
$$

implying that Bob gains no information without the cooperation of Charlie and Diana. Analogous observations hold for the state $\left|\Psi^{-}\right\rangle$in Eqn. (9) and states $\left|\Phi^{ \pm}\right\rangle$in Eqn. (10).

\subsection{Case II: $\left|\psi_{c}\right\rangle$ is 4 -qubit cluster state $\left(\left|C_{4}\right\rangle\right)$}

We assume that Alice has chosen 4-qubit cluster state $\left(\left|C_{4}\right\rangle\right)$ state as the channel and kept the first qubit with her and has sent the second, third and fourth qubits to Bob, Charlie and Diana respectively. In that case

$$
\begin{aligned}
\left|\psi_{c}\right\rangle=\left|C_{4}\right\rangle_{A B C D} & =\frac{1}{2}[|0000\rangle+|0011\rangle+|1100\rangle-|1111\rangle]_{A B C D} \\
& =\frac{1}{\sqrt{2}}\left[|0\rangle_{A}\left|\psi_{0}\right\rangle_{B C D}+|1\rangle_{A}\left|\psi_{1}\right\rangle_{B C D}\right]
\end{aligned}
$$

where $\left|\psi_{0}\right\rangle_{B C D}=\frac{1}{\sqrt{2}}[|000\rangle+|011\rangle]$ and $\left|\psi_{1}\right\rangle_{B C D}=\frac{1}{\sqrt{2}}[|100\rangle-|111\rangle]$.

Now after Alice's Bell measurement on the first two qubits, the combined state of Bob, Charlie and Diana collapses according to Table 1. If Alice's measurement outcome is $\left|\psi^{ \pm}\right\rangle$then the combined state of the agents is

$$
\begin{aligned}
\left|\Psi^{ \pm}\right\rangle_{B C D} & =\frac{1}{\sqrt{1+|\lambda|^{2}}}\left[\left|\psi_{0}\right\rangle_{B C D} \pm \lambda\left|\psi_{1}\right\rangle_{B C D}\right] \\
& =\frac{1}{\sqrt{2\left(1+|\lambda|^{2}\right)}}[|000\rangle+|011\rangle \pm \lambda(|100\rangle-|111\rangle)]_{B C D}
\end{aligned}
$$


Similarly, if Alice obtains $\left|\phi^{ \pm}\right\rangle$then the combined state of the agents is

$$
\begin{aligned}
\left|\Phi^{ \pm}\right\rangle_{B C D} & =\frac{1}{\sqrt{1+|\lambda|^{2}}}\left[\left|\psi_{1}\right\rangle_{B C D} \pm \lambda\left|\psi_{0}\right\rangle_{B C D}\right] \\
& =\frac{1}{\sqrt{2\left(1+|\lambda|^{2}\right)}}[|100\rangle-|111\rangle \pm \lambda(|000\rangle+|011\rangle)]_{B C D}
\end{aligned}
$$

Now from (5), (6), (14) and (15) we can easily observe the following symmetry:

$$
\begin{aligned}
& \left.\left.\left|\Psi^{ \pm}\right\rangle_{B C D}\right|_{\left|\psi_{c}\right\rangle=|\Omega\rangle} \equiv\left|\Psi^{ \pm}\right\rangle_{D C B}\right|_{\left|\psi_{c}\right\rangle=\left|C_{4}\right\rangle}, \\
& \left.\left.\left|\Phi^{ \pm}\right\rangle_{B C D}\right|_{\left|\psi_{c}\right\rangle=|\Omega\rangle} \equiv\left|\Phi^{ \pm}\right\rangle_{D C B}\right|_{\left|\psi_{c}\right\rangle=\left|C_{4}\right\rangle}
\end{aligned}
$$

Thus after the measurement of Alice the combined states of the agents in this case (i.e., when $\left|\psi_{c}\right\rangle=\left|C_{4}\right\rangle$ ) is equivalent to that in the previous case (i.e., when $\left|\psi_{c}\right\rangle=|\Omega\rangle$ ) with the only difference that the role of Diana and Bob are now reversed. Consequently, we obtain a HQIS scheme with $\left|\psi_{c}\right\rangle=\left|C_{4}\right\rangle_{A B C D}$. However, here Bob is more powerful than Charlie and Diana. To be precise, if Diana (Charlie) wishes to reconstruct the unknown quantum state, then Bob and Charlie (Bob and Diana) have to perform a joint measurement on their qubits. However, Bob will be able to reconstruct the state using the measurement outcome of either Charlie or Diana.

The framework used above to obtain these examples of HQIS is quite general and may be used to investigate the possibility of perfect HQIS using larger $n>3$ quantum states. However, we are not interested in that. Rather we are interested to investigate the possibilities of introducing hierarchy (asymmetry) in the other variants of QIS. To be precise, in the following section we will investigate the possibility of probabilistic HQIS and in the subsequent section we will introduce hierarchical quantum secret sharing (HQSS).

\section{A scheme for probabilistic HQIS}

In the previous section we have described a scheme of perfect HQIS using the maximally entangled state. Here we will introduce a scheme of probabilistic HQIS. To do so we assume that Alice prepares and shares with her agents a non-maximally entangled state of the form $\left|\psi_{c^{\prime}}\right\rangle=a|0\rangle\left|\psi_{0}\right\rangle+b|1\rangle\left|\psi_{1}\right\rangle$, where $|a|^{2}+|b|^{2}=1$ and $|a| \neq \frac{1}{\sqrt{2}}$. Now we may follow the previous scheme of perfect teleportation to visualize the difference. Here with the unknown state the initial state of the system would become

$$
\begin{aligned}
\left|\psi_{s}\right\rangle \otimes\left|\psi_{c^{\prime}}\right\rangle & =\frac{1}{\sqrt{1+|\lambda|^{2}}}(|0\rangle+\lambda|1\rangle) \otimes\left[a|0\rangle\left|\psi_{0}\right\rangle+b|1\rangle\left|\psi_{1}\right\rangle\right] \\
& =\frac{1}{\sqrt{\left(1+|\lambda|^{2}\right)}}\left[a|00\rangle\left|\psi_{0}\right\rangle+b|01\rangle\left|\psi_{1}\right\rangle\right]+\frac{\lambda}{\sqrt{\left(1+|\lambda|^{2}\right)}}\left[a|10\rangle\left|\psi_{0}\right\rangle+b|11\rangle\left|\psi_{1}\right\rangle\right] \\
& =\frac{1}{\sqrt{2\left(1+|\lambda|^{2}\right)}}\left[\left|\psi^{+}\right\rangle\left(a\left|\psi_{0}\right\rangle+\lambda b\left|\psi_{1}\right\rangle\right)+\left|\psi^{-}\right\rangle\left(a\left|\psi_{0}\right\rangle-\lambda b\left|\psi_{1}\right\rangle\right)\right. \\
& \left.+\left|\phi^{+}\right\rangle\left(b\left|\psi_{1}\right\rangle+\lambda a\left|\psi_{0}\right\rangle\right)+\left|\phi^{-}\right\rangle\left(b\left|\psi_{1}\right\rangle-\lambda a\left|\psi_{0}\right\rangle\right)\right] .
\end{aligned}
$$

Now Alice does a Bell measurement on the first 2 qubits. Therefore, after the Bell measurement of Alice the combined state of all the agents reduces to $\left|\Psi^{\prime \pm}\right\rangle=\frac{a\left|\psi_{0}\right\rangle \pm \lambda b\left|\psi_{1}\right\rangle}{\sqrt{|a|^{2}+|b \lambda|^{2}}}$ or $\left|\Phi^{\prime \pm}\right\rangle=\frac{b\left|\psi_{1}\right\rangle \pm \lambda a\left|\psi_{0}\right\rangle}{\sqrt{|b|^{2}+|a \lambda|^{2}}}$. To be precise, the relation between the outcome of the Bell measurement of Alice and the combined state of the agents are given in Table 4, which is true in general. But to prove a specific example of probabilistic HQIS let us consider that $\left|\psi_{c^{\prime}}\right\rangle$ is a non-maximally entangled state of $|\Omega\rangle$ type and is described as

$$
\begin{aligned}
\left|\psi_{c^{\prime}}\right\rangle=\left|\Omega^{\prime}\right\rangle_{A B C D} & =[a|0000\rangle+a|0110\rangle+b|1001\rangle-b|1111\rangle]_{A B C D} \\
& =\left[a|0\rangle_{A}\left|\psi_{0}\right\rangle_{B C D}+b|1\rangle_{A}\left|\psi_{1}\right\rangle_{B C D}\right]
\end{aligned}
$$

where $\left|\psi_{0}\right\rangle_{B C D}=\frac{1}{\sqrt{2}}[|000\rangle+|110\rangle],\left|\psi_{1}\right\rangle_{B C D}=\frac{1}{\sqrt{2}}[|001\rangle-|111\rangle],|a|^{2}+|b|^{2}=1$ and $|a| \neq \frac{1}{\sqrt{2}}$ as before. 


\begin{tabular}{|c|c|}
\hline $\begin{array}{c}\text { Outcome of Alice's } \\
\text { measurement }\end{array}$ & $\begin{array}{c}\text { Combined state of all } \\
\text { agents after the } \\
\text { measurement of Alice }\end{array}$ \\
\hline$\left|\psi^{+}\right\rangle$ & $\left|\Psi^{\prime+}\right\rangle=\frac{a\left|\psi_{0}\right\rangle+\lambda b\left|\psi_{1}\right\rangle}{\sqrt{|a|^{2}+|b \lambda|^{2}}}$ \\
\hline$\left|\psi^{-}\right\rangle$ & $\left|\Psi^{\prime-}\right\rangle=\frac{a\left|\psi_{0}\right\rangle-\lambda b\left|\psi_{1}\right\rangle}{\sqrt{|a|^{2}+|b \lambda|^{2}}}$ \\
\hline$\left|\phi^{+}\right\rangle$ & $\left|\Phi^{\prime+}\right\rangle=\frac{b\left|\psi_{1}\right\rangle+\lambda a\left|\psi_{0}\right\rangle}{\sqrt{|b|^{2}+|a \lambda|^{2}}}$ \\
\hline$\left|\phi^{-}\right\rangle$ & $\left|\Phi^{\prime-}\right\rangle=\frac{b\left|\psi_{1}\right\rangle-\lambda a\left|\psi_{0}\right\rangle}{\sqrt{|b|^{2}+|a \lambda|^{2}}}$ \\
\hline
\end{tabular}

Table 4: Relation between Alice's measurement outcomes and the combined states of the agents after the measurement of Alice. Here the quantum channel is non-maximally entangled.

Now in this particular case after Alice's Bell measurement on the first two qubits, the combined state of Bob, Charlie and Diana collapses according to Table 4. If Alice's measurement outcome is $\left|\psi^{ \pm}\right\rangle$then the combined state of the agents is

$$
\begin{aligned}
\left|\Psi^{\prime \pm}\right\rangle_{B C D} & =\frac{1}{\sqrt{|a|^{2}+|b \lambda|^{2}}}\left[a\left|\psi_{0}\right\rangle_{B C D} \pm \lambda b\left|\psi_{1}\right\rangle_{B C D}\right] \\
& =\frac{1}{\sqrt{|a|^{2}+|b \lambda|^{2}}}[a(|000\rangle+|110\rangle) \pm \lambda b(|001\rangle-|111\rangle)]_{B C D}
\end{aligned}
$$

Similarly, if Alice obtains $\left|\phi^{ \pm}\right\rangle$then the state of the agents is

$$
\begin{aligned}
\left|\Phi^{\prime \pm}\right\rangle_{B C D} & =\frac{1}{\sqrt{|b|^{2}+|a \lambda|^{2}}}\left[b\left|\psi_{1}\right\rangle_{B C D} \pm \lambda a\left|\psi_{0}\right\rangle_{B C D}\right] \\
& =\frac{1}{\sqrt{|b|^{2}+|a \lambda|^{2}}}[b(|001\rangle-|111\rangle) \pm \lambda a(|000\rangle+|110\rangle)]_{B C D}
\end{aligned}
$$

Now if the agents decide that Diana will recover the quantum state, then we can decompose (18) and (19) as

$$
\left|\Psi^{\prime \pm}\right\rangle_{B C D}=\frac{1}{\sqrt{|a|^{2}+|b \lambda|^{2}}}\left[|00\rangle_{B C}\left(a|0\rangle_{D} \pm \lambda b|1\rangle_{D}\right)+|11\rangle_{B C}\left(a|0\rangle_{D} \mp \lambda b|1\rangle_{D}\right)\right]
$$

and

$$
\left|\Phi^{\prime \pm}\right\rangle_{B C D}=\frac{1}{\sqrt{|b|^{2}+|a \lambda|^{2}}}\left[|00\rangle_{B C}\left(b|1\rangle_{D} \pm \lambda a|0\rangle_{D}\right)-|11\rangle_{B C}\left(b|1\rangle_{D} \mp \lambda a|0\rangle_{D}\right)\right] .
$$

Now Bob and Charlie measure their respective qubit in computational basis. Alice has already measured her qubits in Bell basis. Up to this point this protocol is similar to the previous protocol of perfect HQIS. But now Diana will not be able to obtain the unknown state just by following the previous protocol of perfect HQIS. For example, if Bob informs that his measurement outcome is $|0\rangle$ and Alice informs that her measurement outcome is $\left|\psi^{+}\right\rangle$. Then Diana's state is $|\psi\rangle_{1}=\frac{a|0\rangle_{D}+\lambda b|1\rangle_{D}}{\sqrt{|a|^{2}+|b \lambda|^{2}}} \neq \frac{1}{\sqrt{1+|\lambda|^{2}}}(|0\rangle+\lambda|1\rangle)_{D}$. Thus Diana cannot follow previous protocol and apply $I$ to reconstruct the unknown state sent by Alice. In fact, Diana cannot construct a single qubit unitary operation to map $\frac{a|0\rangle_{D}+\lambda b|1\rangle_{D}}{\sqrt{|a|^{2}+|b \lambda|^{2}}}$ to $\frac{1}{\sqrt{1+|\lambda|^{2}}}(|0\rangle+\lambda|1\rangle)_{D}$ without the knowledge of $\lambda$. Therefore, Diana has to change her strategy as follows.

Diana prepares an ancilla qubit in $|0\rangle_{A u x i}$ and applies following unitary operation on her qubits (i.e., on the combined system of her existing qubit and ancilla):

$$
U=\left(\begin{array}{cccc}
\frac{b}{a} & \sqrt{1-\frac{b^{2}}{a^{2}}} & 0 & 0 \\
0 & 0 & 0 & -1 \\
0 & 0 & 1 & 0 \\
\sqrt{1-\frac{b^{2}}{a^{2}}} & -\frac{b}{a} & 0 & 0
\end{array}\right) .
$$




\begin{tabular}{|c|c|c|c|c|c|}
\hline $\begin{array}{c}\text { Alice's } \\
\text { measure- } \\
\text { ment } \\
\text { outcome }\end{array}$ & $\begin{array}{c}\text { Bob's } \\
\text { measure- } \\
\text { ment } \\
\text { outcome }\end{array}$ & $\begin{array}{c}\text { Diana's } \\
\text { measure- } \\
\text { ment } \\
\text { outcome }\end{array}$ & $\begin{array}{c}\text { Diana's } \\
\text { state }\end{array}$ & $\begin{array}{c}\text { Operation } \\
\text { applied by } \\
\text { Diana }\end{array}$ & $\begin{array}{c}\text { Final state } \\
\text { of Diana }\end{array}$ \\
\hline$\left|\psi^{+}\right\rangle$ & $|0\rangle$ & $|0\rangle$ & $\frac{|0\rangle+\lambda|1\rangle}{\sqrt{1+|\lambda|^{2}}}$ & $I$ & $\frac{|0\rangle+\lambda|1\rangle}{\sqrt{1+|\lambda|^{2}}}$ \\
\hline$\left|\psi^{-}\right\rangle$ & $|0\rangle$ & $|0\rangle$ & $\frac{|0\rangle-\lambda|1\rangle}{\sqrt{1+|\lambda|^{2}}}$ & $Z$ & $\frac{|0\rangle+\lambda|1\rangle}{\sqrt{1+|\lambda|^{2}}}$ \\
\hline$\left|\phi^{+}\right\rangle$ & $|0\rangle$ & $|0\rangle$ & $\frac{\lambda|0\rangle+|1\rangle}{\sqrt{1+|\lambda|^{2}}}$ & $X$ & $\frac{|0\rangle+\lambda|1\rangle}{\sqrt{1+|\lambda|^{2}}}$ \\
\hline$\left|\phi^{-}\right\rangle$ & $|0\rangle$ & $|0\rangle$ & $-\frac{(\lambda|0\rangle-1\rangle)}{\sqrt{1+|\lambda|^{2}}}$ & $i Y$ & $\frac{|0\rangle+\lambda|1\rangle}{\sqrt{1+|\lambda|^{2}}}$ \\
\hline$\left|\psi^{+}\right\rangle$ & $|1\rangle$ & $|0\rangle$ & $\frac{|0\rangle-\lambda|1\rangle}{\sqrt{1+|\lambda|^{2}}}$ & $Z$ & $\frac{|0\rangle+\lambda|1\rangle}{\sqrt{1+|\lambda|^{2}}}$ \\
\hline$\left|\psi^{-}\right\rangle$ & $|1\rangle$ & $|0\rangle$ & $\frac{|0\rangle+\lambda|1\rangle}{\sqrt{1+|\lambda|^{2}}}$ & $I$ & $\frac{|0\rangle+\lambda|1\rangle}{\sqrt{1+|\lambda|^{2}}}$ \\
\hline$\left|\phi^{+}\right\rangle$ & $|1\rangle$ & $|0\rangle$ & $\frac{\lambda|0\rangle-|1\rangle}{\sqrt{1+|\lambda|^{2}}}$ & $i Y$ & $\frac{|0\rangle+\lambda|1\rangle}{\sqrt{1+|\lambda|^{2}}}$ \\
\hline$\left|\phi^{-}\right\rangle$ & $|1\rangle$ & $|0\rangle$ & $-\frac{\lambda|0\rangle+1\rangle)}{\sqrt{1+|\lambda|^{2}}}$ & $X$ & $\frac{|0\rangle+\lambda|1\rangle}{\sqrt{1+|\lambda|^{2}}}$ \\
\hline
\end{tabular}

Table 5: Probabilistic HQIS (Diana reconstructs the unknown state): Relation among Alice's measurement outcomes, Bob's (Charlie's) measurement outcomes, Diana's measurement outcomes, unitary operations used by Diana and Diana's conclusions/final state. In the remaining 8 cases where Diana's measurement outcome is $|1\rangle$, her final state will be $|1\rangle$ and the HQIS scheme will fail. Global phases are neglected in the last column.

As $a$ and $b$ are known, construction of $U$ is allowed. Now in the specific case considered above (where Alice's measurement outcome is $\left|\psi^{+}\right\rangle$and that of Bob is $\left.|0\rangle\right)$, Diana applies $U$ on her product state

$$
|\psi\rangle_{2}=|\psi\rangle_{1}|0\rangle_{\text {Auxi }}=\left(\frac{a|0\rangle_{D}+\lambda b|1\rangle_{D}}{\sqrt{|a|^{2}+|b \lambda|^{2}}}\right)|0\rangle_{\text {Auxi }}=\frac{1}{\sqrt{|a|^{2}+|b \lambda|^{2}}}\left(\begin{array}{c}
a \\
0 \\
b \lambda \\
0
\end{array}\right)
$$

and obtains

$$
\begin{aligned}
U|\psi\rangle_{2} & =\frac{1}{\sqrt{|a|^{2}+|b \lambda|^{2}}}\left(\begin{array}{c}
b \\
0 \\
b \lambda \\
\sqrt{1-\frac{b^{2}}{a^{2}}} a
\end{array}\right) \\
& =\frac{1}{\sqrt{|a|^{2}+|b \lambda|^{2}}}\left(b(|0\rangle+\lambda|1\rangle)|0\rangle+\sqrt{a^{2}-b^{2}}|1\rangle|1\rangle\right) .
\end{aligned}
$$

Now Diana measures the last qubit (ancilla) in the computational basis. If her measurement yields $|0\rangle$ then she obtains unknown state with unit fidelity but if her measurement on ancilla yields $|1\rangle$ then the HQIS scheme fails. Similarly, we can check the other 15 possibilities. The complete table that relates Alice's measurement outcomes, Bob's (Charlie's) measurement outcomes, Diana's measurement outcomes and unitary operations to be applied by Diana and Diana's conclusions are given in the Table 5 .

Now consider that Bob recovers the state. In that case we can decompose (18) and (19) as

$$
\begin{aligned}
\left|\Psi^{\prime \pm}\right\rangle_{B C D} & =\frac{1}{\sqrt{2\left(|a|^{2}+|\lambda b|^{2}\right)}}\left[\left(a|0\rangle_{B} \mp \lambda b|1\rangle_{B}\right)\left|\psi^{+}\right\rangle_{C D}+\left(a|0\rangle_{B} \pm \lambda b|1\rangle_{B}\right)\left|\psi^{-}\right\rangle_{C D}\right. \\
& \left.+\left(a|1\rangle_{B} \pm \lambda b|0\rangle_{B}\right)\left|\phi^{+}\right\rangle_{C D}-\left(a|1\rangle_{B} \mp \lambda b|0\rangle_{B}\right)\left|\phi^{-}\right\rangle_{C D}\right],
\end{aligned}
$$




$$
\begin{aligned}
\left|\Phi^{\prime \pm}\right\rangle_{B C D} & =\frac{1}{\sqrt{2\left(|\lambda a|^{2}+|b|^{2}\right)}}\left[\left(b|0\rangle_{B} \pm \lambda a|1\rangle_{B}\right)\left|\phi^{+}\right\rangle_{C D}+\left(b|0\rangle_{B} \mp \lambda a|1\rangle_{B}\right)\left|\phi^{-}\right\rangle_{C D}\right. \\
& \left.-\left(b|1\rangle_{B} \mp \lambda a|0\rangle_{B}\right)\left|\psi^{+}\right\rangle_{C D}+\left(b|1\rangle_{B} \pm \lambda a|0\rangle_{B}\right)\left|\psi^{-}\right\rangle_{C D}\right] .
\end{aligned}
$$

Bob prepares an ancilla qubit in $|0\rangle_{\text {Auxi }}$ and applies the 2-qubit unitary operator $U$ described by (22) on his qubits (i.e., on the combined system of his existing qubit and ancilla) iff the measurement outcome of combined measurement of Charlie and Diana is $\left|\psi^{ \pm}\right\rangle$, otherwise he applies

$$
U_{1}=U(X \otimes I)=\left(\begin{array}{cccc}
0 & 0 & \frac{b}{a} & \sqrt{1-\frac{b^{2}}{a^{2}}} \\
0 & -1 & 0 & 0 \\
1 & 0 & 0 & 0 \\
0 & 0 & \sqrt{1-\frac{b^{2}}{a^{2}}} & -\frac{b}{a}
\end{array}\right)
$$

on his qubits. To be precise, if the outcome of joint measurement of Charlie and Diana is $\left|\psi^{ \pm}\right\rangle\left(\left|\phi^{ \pm}\right\rangle\right)$then Bob applies $U\left(U_{1}\right)$ on his qubits. Subsequently he measures the auxiliary qubit. If his measurement on the auxiliary qubit yields $|1\rangle$ then the scheme fails otherwise he recovers the unknown state by applying appropriate unitary operations. The relation among the measurement outcomes of Alice, outcomes of joint measurement of Charlie and Diana, measurement outcomes of Bob, unitary operations used by Bob and Bob's conclusions/final state is shown in the Table 6. Clearly Bob needs help of both Charlie and Diana to reconstruct the state sent by Alice as before.

Thus using this scheme Alice can hierarchically split quantum information among her agents and one of the agents can recover the unknown quantum state with unit fidelity. However, the success rate of the scheme is not unity. This is why it can be referred to as probabilistic HQIS in analogy with the conventional probabilistic teleportation scheme.

\section{Hierarchical quantum secret sharing (HQSS)}

The QSS scheme was originally proposed in 1999 [12]. The scheme may now be generalized and modified as follows. As before consider that Alice is boss of a company and Bob, Charlie and Diana are her agents. Further, consider that Alice lives in Amsterdam. Bob, Charlie and Diana are her agents in Berlin. Alice wants to send them a secret message to perform a job. However, one of them may be dishonest and Alice does not know who is dishonest. But Diana is a senior employee of the company and she has been working in the company since long, while Bob and Charlie has joined recently. So Alice trusts Diana more than the other two agents. Thus there is a hierarchy among the agents. In this situation, Alice may use HQIS scheme with 4-qubit $|\Omega\rangle$ as described in Subsection 2.1 and send the information in three pieces so that none of Bob, Charlie and Diana can read the message of Alice without the help of the others. However, Diana would require lesser help than Bob. Now there exist possibilities of eavesdropping. For example, consider that Bob is dishonest and he captures the qubit sent to Charlie and Diana too. If Bob does a Bell measurement on Charlie's and Diana's qubit then using the unitary operations described in Table 3 , he will be able to get the entire information without any help of Charlie and Diana. Consequently, to circumvent this kind of attack from a dishonest user or an external eavesdropper, Alice needs to add some error checking schemes to the above proposed HQIS schemes. One way to achieve this is as follows:

HQSS 1: Alice prepares $|\Omega\rangle^{\otimes n}$. As $|\Omega\rangle^{\otimes n}$ is a $4 n$-qubit state, qubits of $|\Omega\rangle^{\otimes n}$ may be indexed as $p_{1}, p_{2}, \cdots, p_{4 n}$. Thus $p_{s}$ is the $s^{\text {th }}$ qubit of $|\Omega\rangle^{\otimes n}$ and $\left\{p_{4 l-3}, p_{4 l-2}, p_{4 l-1}, p_{4 l}: l \leq n\right\}$ are the 4 qubits of the $l^{\text {th }}$ copy of $|\Omega\rangle^{\otimes n}$. 


\begin{tabular}{|c|c|c|c|c|c|c|}
\hline $\begin{array}{c}\text { Alice's mea- } \\
\text { surement } \\
\text { outcome }\end{array}$ & $\begin{array}{c}\text { Outcome of } \\
\text { joint mea- } \\
\text { surement of } \\
\text { Charlie and } \\
\text { Diana } \\
\end{array}$ & $\begin{array}{c}\text { Bob's two } \\
\text { qubit } \\
\text { operation }\end{array}$ & $\begin{array}{c}\text { Bob's mea- } \\
\text { surement } \\
\text { outcome }\end{array}$ & Bob's state & $\begin{array}{c}\text { Bob's } \\
\text { operation }\end{array}$ & $\begin{array}{c}\text { Final state } \\
\text { of Bob }\end{array}$ \\
\hline$\left|\psi^{+}\right\rangle$ & $\left|\psi^{+}\right\rangle_{C D}$ & $\bar{U}$ & $|0\rangle$ & $\frac{|0\rangle-\lambda|1\rangle}{\sqrt{1+|\lambda|^{2}}}$ & $Z$ & $\frac{|0\rangle+\lambda|1\rangle}{\sqrt{1+|\lambda|^{2}}}$ \\
\hline$\left|\psi^{+}\right\rangle$ & $\left|\psi^{-}\right\rangle_{C D}$ & $U$ & $|0\rangle$ & $\frac{|0\rangle+\lambda|1\rangle}{\sqrt{1+|\lambda|^{2}}}$ & $I$ & $\frac{|0\rangle+\lambda|1\rangle}{\sqrt{1+|\lambda|^{2}}}$ \\
\hline$\left|\psi^{-}\right\rangle$ & $\left|\psi^{+}\right\rangle_{C D}$ & $\bar{U}$ & $\overline{|0\rangle}$ & $\frac{|0\rangle+\lambda|1\rangle}{\sqrt{1+|\lambda|^{2}}}$ & $I$ & $\frac{|0\rangle+\lambda|1\rangle}{\sqrt{1+|\lambda|^{2}}}$ \\
\hline$\overline{\left|\psi^{-}\right\rangle}$ & $\left|\psi^{-}\right\rangle_{C D}$ & $\bar{U}$ & $|0\rangle$ & $\frac{|0\rangle-\lambda|1\rangle}{\sqrt{1+|\lambda|^{2}}}$ & $Z$ & $\frac{|0\rangle+\lambda|1\rangle}{\sqrt{1+|\lambda|^{2}}}$ \\
\hline$\left|\phi^{+}\right\rangle$ & $\left|\psi^{+}\right\rangle_{C D}$ & $U$ & $|0\rangle$ & $-\frac{(|1\rangle-\lambda|0\rangle)}{\sqrt{1+|\lambda|^{2}}}$ & $X Z$ & $\frac{|0\rangle+\lambda|1\rangle}{\sqrt{1+|\lambda|^{2}}}$ \\
\hline$\left|\phi^{+}\right\rangle$ & $\left|\psi^{-}\right\rangle_{C D}$ & $U$ & $|0\rangle$ & $\frac{|1\rangle+\lambda|0\rangle}{\sqrt{1+|\lambda|^{2}}}$ & $X$ & $\frac{|0\rangle+\lambda|1\rangle}{\sqrt{1+|\lambda|^{2}}}$ \\
\hline$\left|\phi^{-}\right\rangle$ & $\left|\psi^{+}\right\rangle_{C D}$ & $U$ & $|0\rangle$ & $-\frac{(|1\rangle+\lambda|0\rangle)}{\sqrt{1+|\lambda|^{2}}}$ & $X$ & $\frac{|0\rangle+\lambda|1\rangle}{\sqrt{1+|\lambda|^{2}}}$ \\
\hline$\left|\phi^{-}\right\rangle$ & $\left|\psi^{-}\right\rangle_{C D}$ & $\overline{U_{1}}$ & $|0\rangle$ & $\frac{|1\rangle-\lambda|0\rangle}{\sqrt{1+|\lambda|^{2}}}$ & $X Z$ & $\frac{|0\rangle+\lambda|1\rangle}{\sqrt{1+|\lambda|^{2}}}$ \\
\hline$\left|\psi^{+}\right\rangle$ & $\left|\phi^{+}\right\rangle_{C D}$ & $U_{1}$ & $|0\rangle$ & $\frac{|0\rangle+\lambda|1\rangle}{\sqrt{1+|\lambda|^{2}}}$ & $I$ & $\frac{|0\rangle+\lambda|1\rangle}{\sqrt{1+|\lambda|^{2}}}$ \\
\hline$\overline{\left|\psi^{+}\right\rangle}$ & $\left|\phi^{-}\right\rangle_{C D}$ & $\overline{U_{1}}$ & $|0\rangle$ & $-\frac{(|0\rangle-\lambda|1\rangle)}{\sqrt{1+|\lambda|^{2}}}$ & $Z$ & $\frac{|0\rangle+\lambda|1\rangle}{\sqrt{1+|\lambda|^{2}}}$ \\
\hline$\left|\psi^{-}\right\rangle$ & $\left|\phi^{+}\right\rangle_{C D}$ & $\overline{U_{1}}$ & $|0\rangle$ & $\frac{|0\rangle-\lambda|1\rangle}{\sqrt{1+|\lambda|^{2}}}$ & $Z$ & $\frac{|0\rangle+\lambda|1\rangle}{\sqrt{1+|\lambda|^{2}}}$ \\
\hline$\left|\psi^{-}\right\rangle$ & $\left|\phi^{-}\right\rangle_{C D}$ & $\overline{U_{1}}$ & $|0\rangle$ & $-\frac{(|0\rangle+\lambda|1\rangle)}{\sqrt{1+|\lambda|^{2}}}$ & $I$ & $\frac{|0\rangle+\lambda|1\rangle}{\sqrt{1+|\lambda|^{2}}}$ \\
\hline$\left|\phi^{+}\right\rangle$ & $\left|\phi^{+}\right\rangle_{C D}$ & $U_{1}$ & $|0\rangle$ & $\frac{|1\rangle+\lambda|0\rangle}{\sqrt{1+|\lambda|^{2}}}$ & $X$ & $\frac{|0\rangle+\lambda|1\rangle}{\sqrt{1+|\lambda|^{2}}}$ \\
\hline$\left|\phi^{+}\right\rangle$ & $\left|\phi^{-}\right\rangle_{C D}$ & $\overline{U_{1}}$ & $|0\rangle$ & $\frac{|1\rangle-\lambda|0\rangle}{\sqrt{1+|\lambda|^{2}}}$ & $X Z$ & $\frac{|0\rangle+\lambda|1\rangle}{\sqrt{1+|\lambda|^{2}}}$ \\
\hline$\left|\phi^{-}\right\rangle$ & $\left|\phi^{+}\right\rangle_{C D}$ & $\overline{U_{1}}$ & $|0\rangle$ & $\frac{|1\rangle-\lambda|0\rangle}{\sqrt{1+|\lambda|^{2}}}$ & $X Z$ & $\frac{|0\rangle+\lambda|1\rangle}{\sqrt{1+|\lambda|^{2}}}$ \\
\hline$\left|\phi^{-}\right\rangle$ & $\left|\phi^{-}\right\rangle_{C D}$ & $\overline{U_{1}}$ & $\overline{|0\rangle}$ & $\frac{|1\rangle+\lambda|0\rangle}{\sqrt{1+|\lambda|^{2}}}$ & $X$ & $\frac{|0\rangle+\lambda|1\rangle}{\sqrt{1+|\lambda|^{2}}}$ \\
\hline
\end{tabular}

Table 6: Probabilistic HQIS (Bob reconstructs the unknown state): The relation among the measurement outcomes of Alice, outcomes of joint measurement of Charlie and Diana, measurement outcomes of Bob, unitary operations used by Bob and Bob's conclusions/final state. In the remaining 16 cases where Bob's measurement outcome is $|1\rangle$, his final state will be $|1\rangle$ and the HQIS scheme will fail. Global phases are ignored in the last column. 
HQSS 2: Using all the first qubits of her possession, Alice creates an ordered sequence $P_{A}=\left[p_{1}, p_{5}, p_{9}, \cdots, p_{4 n-3}\right]$. Similarly she prepares an ordered sequence with all the second qubits as $P_{B}=\left[p_{2}, p_{6}, p_{10}, \cdots, p_{4 n-2}\right]$, another ordered sequence with all the third qubits as $P_{C}=\left[p_{3}, p_{7}, p_{11}, \cdots, p_{4 n-1}\right]$ and another ordered sequence with all the fourth qubits as $P_{D}=\left[p_{4}, p_{8}, p_{12}, \cdots, p_{4 n}\right]$. She prepares $3 n$ decoy qubits $d_{i}$ with $i=1,2, \cdots, 3 n$ such that $d_{i} \in\{|0\rangle,|1\rangle,|+\rangle,|-\rangle\}$ and concatenates first $n$ of them with $P_{B}$ to yield a larger sequence $P_{B^{\prime}}=\left[p_{2}, p_{6}, p_{10}, \cdots, p_{4 n-2}, d_{1}, d_{2}, \cdots, d_{n}\right]$. Similarly, using $P_{C}$ and next $n$ decoy qubits she creates $P_{C^{\prime}}=\left[p_{3}, p_{7}, p_{11}, \cdots, p_{4 n-1}, d_{n+1}, d_{n+2}, \cdots, d_{2 n}\right]$ and using $P_{D}$ and the last $n$ decoy qubits she creates $P_{D^{\prime}}=\left[p_{4}, p_{8}, p_{12}, \cdots, p_{4 n}, d_{2 n+1}, d_{2 n+2}, \cdots, d_{3 n}\right]$. Thereafter Alice applies a permutation operator $\Pi_{2 n}$ on $P_{B^{\prime}}, P_{C^{\prime}}$ and $P_{D^{\prime}}$ to create random sequences $P_{B^{\prime \prime}}=\Pi_{2 n} P_{B^{\prime}}, P_{C^{\prime \prime}}=\Pi_{2 n} P_{C^{\prime}}$ and $P_{D^{\prime \prime}}=\Pi_{2 n} P_{D^{\prime}}$ and sends $P_{B^{\prime \prime}}, P_{C^{\prime \prime}}$ and $P_{D^{\prime \prime}}$ to Bob, Charlie and Diana respectively. The actual order is known to Alice only.

HQSS 3: Alice announces $\Pi_{n} \in \Pi_{2 n}$, the coordinates of the decoy qubits in each sequence after receiving authenticated acknowledgments of receipt of all the qubits form Bob, Charlie and Diana. The BB84 subroutine 4 to detect eavesdropping, is then implemented on the decoy qubits by measuring them in the nonorthogonal bases $\{|0\rangle,|1\rangle\}$ or $\{|+\rangle,|-\rangle\}$. If sufficiently few errors are found in all the sequences, then they go to the next step; else, they return to HQSS 1.

This will ensure that the initial $|\Omega\rangle^{\otimes n}$ state is appropriately distributed among Alice, Bob, Charlie and Diana without any eavesdropping. As the eavesdropping is checked by the BB84 subroutine, security of the protocol is equivalent to that of BB84 protocol.

HQSS 4: Alice discloses the coordinates of the remaining qubits and Bob, Charlie and Diana rearrange their sequences accordingly.

Remaining part of the protocol is same as HQIS scheme described in Subsection 2.1. Now consider that Alice's quantum secret which is to be shared is $\left|\psi_{s}\right\rangle=\frac{1}{\sqrt{1+|\lambda|^{2}}}(|0\rangle+\lambda|1\rangle)$. Thus Alice, Bob, Charlie and Diana share $n$ 5-qubit states of the form (3), where first two qubits are with Alice and the last three qubits are with Bob, Charlie and Diana, and $\left|\psi_{0}\right\rangle_{B C D}=\frac{1}{\sqrt{2}}[|000\rangle+|110\rangle]$ and $\left|\psi_{1}\right\rangle_{B C D}=\frac{1}{\sqrt{2}}[|001\rangle-|111\rangle]$.

HQSS 5: Alice measures her qubits in Bell basis and announces the results.

Without loss of generalization we may assume that Alice has asked Bob to prepare the secret state transmitted by her.

HQSS 6: Charlie and Diana jointly measures their qubits using Bell basis and communicate the result to Bob.

HQSS 7: Bob applies appropriate unitary operators (as described in Table 3) in accordance with the measurement outcomes of Alice, Charlie and Diana, and reconstructs the secret quantum state transmitted by Alice.

If Alice asked Diana to prepare the secret state transmitted by her, then the last two steps should be modified as follows:

\footnotetext{
${ }^{4}$ BB84 subroutine means eavesdropping is checked using conjugate coding in a manner similar to what followed in BB84 protocol. Explicitly, in our particular case, Alice's announcement of the position of the decoy qubits provides a verification string to each of her agents. Now a agent measures either all the qubits of the verification string randomly in $\{0,1\}$ or $\{+,-\}$ basis and announces which basis she (he) has used to measure a particular qubit, position of that qubit in the string and outcome. Alice compares the initial states of the decoy qubits with the outcomes of an agent in all those cases where the basis used by her to prepare the decoy qubit is same as the basis used by the particular agent to measure it. Ideally in absence of eavesdropping it should match. Any eavesdropping effort would lead to mismatch.
} 
HQSS 6: Bob (Charlie) measures his qubit using computational basis and communicates the result to Diana.

HQSS 7: Diana applies appropriate unitary operators (as described in Table 2) in accordance with the measurement outcomes of Alice and Bob (Charlie), and reconstructs the secret quantum state transmitted by Alice.

This is clearly a scheme of HQSS as Diana requires lesser information than Bob to obtain the secret shared by Alice. This provides a clear and unconditionally secure solution to the practical problem described in the beginning of this section where Alice wishes to distribute a quantum state among her agents in an asymmetric and secure manner. It also provides a solution to the practically relevant problem related to the use of nuclear weapon described in Section 1. Here it would be apt to note that we have explicitly described a scheme for HQSS using $|\Omega\rangle$ state. However, the protocol described above is much more general and any quantum state where HQIS is possible may be used to implement HQSS. For example, 4-qubit cluster state, 6-qubit cluster state and graph state can be used to implement HQSS. Further, it is now a straightforward task to turn this HQSS scheme into a probabilistic HQSS scheme. In the earlier studies of Wang et al. possibility of HQSS was hinted but neither an explicit protocol was provided nor the security threats arising from one of the user being dishonest was properly discussed. Thus the present scheme is the first ever scheme of HQSS which is expected to find applications in many important practical situations.

\section{Conclusions}

We have generalized Wang et al.'s idea of asymmetric quantum information splitting from various perspectives. To be precise, we have provided a more general framework to study the hierarchical quantum information splitting (HQIS) and have shown that the same can be modified to yield protocols of HQSS and probabilistic HQIS. The generalization is important for several reasons. Firstly, there exist several practical situations where the asymmetric information splitting (Especially HQSS) is relevant. Secondly, the possibility of HQSS and probabilistic HQIS were not investigated earlier. Following a similar line of arguments we can also obtain a scheme for hierarchical joint remote state preparation. That would be shown elsewhere [16]. Further the approach adopted in the present paper can be easily used to explore the possibilities of observing HQIS, probabilistic HQIS and HQSS in other quantum states.

Acknowledgment: A. P. thanks Department of Science and Technology (DST), India for support provided through the DST project No. SR/S2/LOP-0012/2010. He also thanks the Operational Program Education for Competitiveness - European Social Fund project CZ.1.07/2.3.00/20.0017 of the Ministry of Education, Youth and Sports of the Czech Republic. Authors also thank Dr. R. Srikanth for some helpful technical discussions.

\section{References}

[1] C.H. Bennett. G. Brassard, C. Crépeau, R. Jozsa, A. Peres and W. K. Wootters, Phys. Rev. Lett., 70 (1993) 1895.

[2] A. Pathak and A. Banerjee, , Int. J. Quan. Info. 9 (2011) 389.

[3] W.-L. Li, C.-F. Li and G.-C Guo, Phys. Rev. A 61 (2000) 034301.

[4] Z.-L. Cao, M. Yang, G.-C. Guo, Phys. Lett. A 308 (2003) 349.

[5] H. Lu, G.-C. Guo, Phys. Lett. A 276 (2000) 209. 
[6] B.-S. Shi, Y.-K. Jiang, G.-C. Guo, Phys. Lett. A 268 (2000) 161.

[7] S. Muralidharan and P. K. Panigrahi, Phys. Rev. A 78 (2008) 062333.

[8] P. Agrawal and A. Pati, Phys. Rev. 74 (2006) 062320.

[9] X.-W. Wang, L.-X. Xiac, Z.-Y. Wangd, D.-Y. Zhang, Opt. Comm. 283 (2010) 1196.

[10] X.-W. Wang, D.-Y. Zhang, S.-Q. Tang and L.-J. Xie, J. Phys. B 44 (2011) 035505.

[11] X.-W. Wang, D.-Y. Zhang, S.-Q. Tang, X.-G. Zhan, K.-M.You, Int. J. Theor. Phys. 49 (2010) 2691.

[12] M. Hillery, V. Buzek and A. Bertaiume, Phys. Rev. A 59 (1999) 1829.

[13] A.K. Pati, Phys. Rev. A 63 (2000) 014302.

[14] N.B. An and J. Kim, J. Phys. B. 41 (2008) 095501.

[15] B. Pradhan, P. Agrawal and A.K. Pati, arXiv:0705.1917v1 (quant-ph).

[16] C. Shukla and A. Pathak, Hierarchial joint remote state preparation, under preparation. 